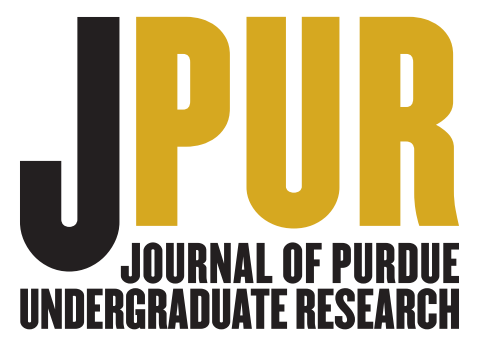

\title{
SSLC
}

\section{Big Data Visualization: HoloLens Brings Meaningful Interaction to Lupus Medical Data}

\section{Student researcher: Dylan Martin, Sophomore}

Scientists often deal with large amounts of data when conducting experiments, and statisticians often find that the data goes mostly unused. A good data scientist's goal is to seek out ways to display impactful analyses about data sets that can easily inform any individual. This research explores how augmented reality devices, specifically the Microsoft HoloLens, can provide a brand-new space to uniquely visualize data.

The HoloLens' augmented reality allows a user to see their surroundings, as well as the holograms they choose to display. This means that a user can manipulate and interact with holograms in their environment in many different ways, including voice, gaze, and physical gestures. The focus of the research is developing a unique application for the HoloLens that allows users to recreate 2D graphs, changing them into interactive $3 \mathrm{D}$ visualizations.

In the future, this research will allow any individual, including those with no scientific or technological background, to be able to easily interact with and understand various types of data. This eliminates the learning curve often associated with scientific fields, and allows anyone to have the data manipulation skills that were once restricted to those with formal training or higher education. The importance of this research stems from the fact that data exists in every corner of society. Using data can help solve real-world problems more efficiently and effectively.

Byrd writes: "Data visualization wrapped in augmented/ hybrid reality is allowing researchers and scientists a new level of engagement with their data. Dylan is creating an environment to explore complex lupus data. His progress in this area will enable new insights into the data, detection, and treatment of the disease."

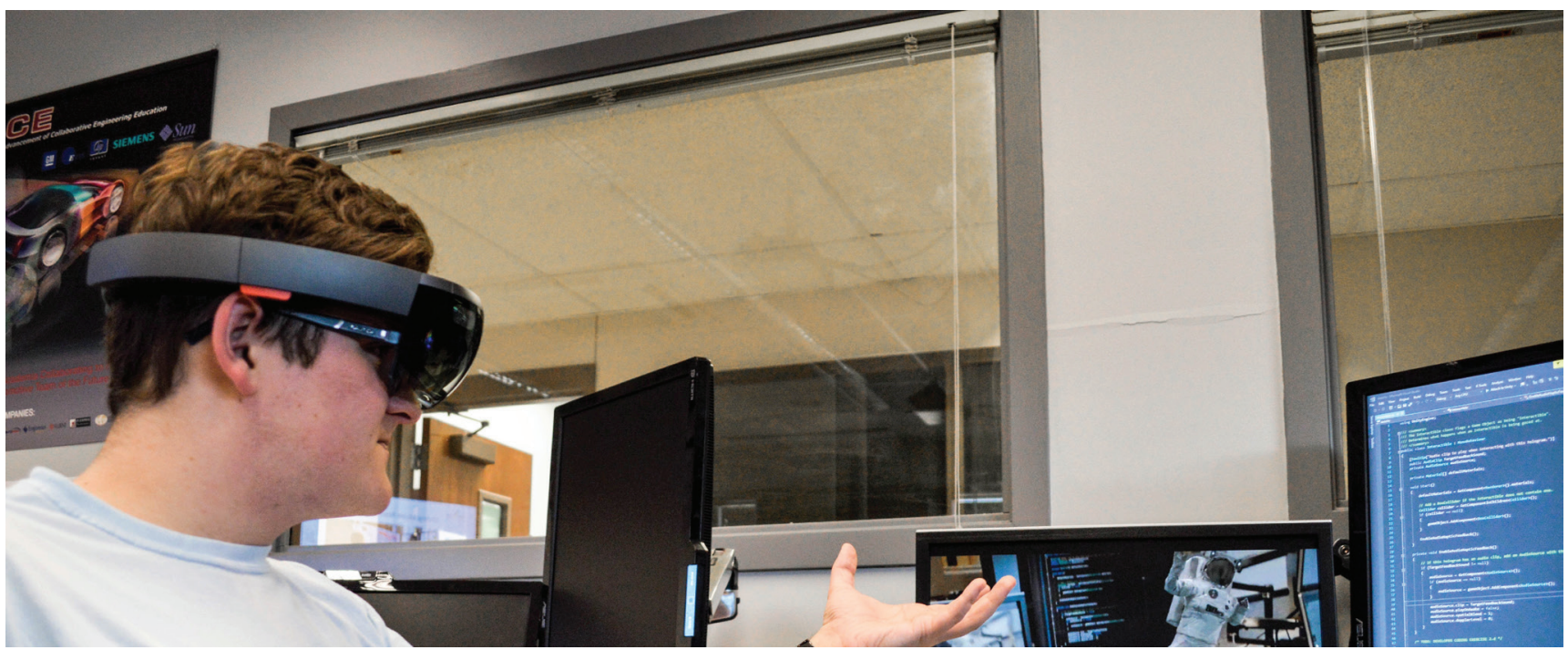

HoloLens enables Martin to see his environment, but also any holograms he chooses to place within.

Martin, D. (2018). Big data visualization: HoloLens brings meaningful interaction to lupus medical data. Journal of Purdue Undergraduate Research, 8, 85-86. https://doi.org/10.5703/1288284316759 